\title{
Cuarenta años del PREVI-LIMA: Algunas enseñanzas para la industrialización de la vivienda de bajo coste en Latinoamérica
}

\author{
PREVI-LIMA forty years later: teachings for industrialised \\ low-cost housing in Latin America
}

P. Lucas ${ }^{(*)}$, L. Salas ${ }^{(*)}$, R. Barrionuevo(**)

RESUMEN

EI PREVI, Proyecto Experimental de Vivienda, comenzó a gestarse en Lima en el año 1967 y entre otras iniciativas, puso en marcha un concurso internacional de arquitectura para la construcción de un barrio de unas 500 viviendas basado en las propuestas de equipos como Atelier 5, Aldo van Eyck o Íñiguez de Ozoño y Vázquez de Castro. Los cuarenta años transcurridos, así como la continua transformación de las viviendas por sus usuarios, hacen posible reflexionar sobre la adecuación de las distintas tecnologías constructivas propuestas en el concurso.

En esa época se confiaba en la industrialización pesada, como se constata en la propuesta de los arquitectos alemanes o polacos. Sin embargo, bastantes propuestas del PREVI optaron por la racionalización constructiva y la fabricación seriada de pequeños elementos, lejos de los grandes prefabricados tridimensionales. La situación en Latinoamérica invita a cuestionar si algunas de las propuestas tecnológicas del PREVI serían viables actualmente.

195-18

Palabras clave: PREVI, vivienda, racionalización, industrialización, Latinoamérica.

\section{SUMMARY}

The PREVI, Spanish initials for "experimental housing project", was conceived in Lima in 1967. Among other initiatives, it launched an international architectural competition that led to the construction of a 500-unit compound based on proposals put forward by teams such as Atelier 5, Aldo van Eyck, and Íniguez de Ozoño and Vázquez de Castro. The forty years that have lapsed in the interim and the ongoing transformation of the homes by their dwellers afford an opportunity to reflect on the suitability of the construction technologies proposed in the competition.

At the time, the tendency was to rely on large-scale industrialisation, as can be seen in the German and Polish architects' proposals. Nonetheless, many of the PREVI proposals opted for rationalising construction and precasting series of small elements, rather than huge threedimensional members. In the situation presently prevailing in Latin America, the viability of some of the technological proposals deployed in the PREVI might be profitably revisited.

Keywords: PREVI, housing, rationalisation, industrialisation, Latin America.

(*) Instituto de Ciencias de la Construcción Eduardo Torroja (CSIC). Madrid (España)

$(* *)$ Universidad Nacional de Ingeniería. Lima (Perú)

Fecha de recepción: 28-02-11

Persona de contacto/Corresponding author: julian.salas@ietcc.csic.es (J. Salas)

Fecha de aceptación: 15-06-11 


\section{1. ¿QUÉ FUE PREVI EN 1970?}

Frente al contexto europeo, en el que el espacio urbano se planifica y construye de manera formal; la situación latinoamericana se caracteriza aún hoy por el predominio de procesos informales. La existencia de numerosos asentamientos no planificados, así como los procesos de auto-construcción de muy diverso tipo, generan un contexto social, técnico y constructivo, que es preciso considerar al plantear soluciones posibles de habitabilidad que empleen tecnologías industrializadas de coste reducido.

¿Qué opciones ofrece la industrialización en estos ámbitos en los que lo informal convive con lo planificado? ¿Cómo responde la industrialización en un entorno en el que es más que probable que se produzcan procesos de autoconstrucción? El PREVI: Proyecto Experimental de Vivienda, realizado en Lima hace cuatro décadas, proporciona algunas claves sobre la acogida e interpretación que los usuarios de las viviendas planteadas han realizado. El hecho de que la mayoría de las propuestas presentadas al concurso hayan sido construidas, unido a la mención explícita que se hacía en las bases de la convocatoria sobre el empleo de procesos industrializados, han hecho del barrio PREVI un banco de pruebas que permite extraer algunas conclusiones. Con el paso del tiempo (Figura 1), y a la vista de las transformaciones que sobre los proyectos han llevado a cabo los usuarios, parece posible evaluar las distintas respuestas que se ejecutaron en este concurso de vivienda social latinoamericana.

El PREVI comenzó a gestarse en 1967, se trataba de una propuesta puesta en marcha por el gobierno peruano con el fin de trazar un plan de mejora de la situación de la vivienda en Lima. A petición de las Naciones Unidas, se realizó un estudio previo para determinar si la problemática de la vivienda local era representativa de la situación de los países en vías de desarrollo, y ante la respuesta afirmativa, el programa contó con la participación de Naciones Unidas. El PREVI en su versión inicial constaba de tres planes o programas de vivienda, denominados proyectos piloto en las bases del concurso, y cuyos objetivos eran los siguientes:

-El PP1 se proponía la construcción de un barrio de nueva planta a partir de las propuestas surgidas de un concurso de ámbito nacional, al que se presentaron 28 propuestas peruanas, e internacional, en el que tomaron parte 13 equipos extranjeros. Para participar en esta sección, Peter Land, arquitecto británico vinculado a la organización, eligió una serie de equipos entre los que se encontraban algunos de los arquitectos más relevantes de aquellos años. Fueron invitados a participar en la sección internacional de PREVI: Atelier 5 (Suiza), Aldo van Eyck (Holanda), Christopher Alexander (USA), Candilis, Josic and Woods (Francia), José Luis Íñiguez de Ozono y Vázquez de Castro (España), James Stirling (Reino Unido), Toivo Korhonen (Finlandia), Germán Samper (Colombia), Fumihiko Maki, Kionori Kikutake (Japón), Charles Correa (India), Herbert Ohl (Alemania), Knud Svenssons (Dinamarca), Oskar Hansen y Svein Hatloy (Polonia).

-El PP2 consistía en la elaboración de un estudio con planes prácticos para la rehabilitación y renovación urbana de áreas degradadas de la ciudad.

-EI PP3 tenía como objetivo la realización de un proyecto de viviendas de bajo coste mediante procesos de autoconstrucción, aportando la asistencia técnica que fuera necesaria.

El fuerte terremoto que asoló Lima el 30 de mayo de 1970 llevó finalmente al desdoblamiento del PP3 en dos programas, uno que continuaba con las ideas de autoconstrucción del PP3 y otro que trabajaba con propuestas sismorresistentes y que pasó a denominarse PP4. El PP4 surge como la búsqueda de soluciones al problema de los damnificados en los desastres naturales: sismos, incendios o aluviones.

Si atendemos a la nómina de participantes de la sección internacional del PP1, nos encontramos con algunos de los nombres más destacados de lo que ha venido denominándose tercera generación del movimiento moderno. Se trata de un grupo de arquitectos que, aunque empiezan a cuestionar algunos de los postulados surgidos en las primeras décadas del siglo, mantienen buena parte de sus intenciones, como demuestra su preocupación sobre el tema de la vivienda social. A fecha de hoy, Charles Correa, uno de los arquitectos invitados a concursar en PREVI, reflexiona así sobre este hecho: Por supuesto, ya desde los inicios del siglo XX los arquitectos se habían implicado en la vivienda social. Fue el motor que hizo funcionar al movimiento moderno, el que desencadenó las preocupaciones -y la imaginación- de Walter Gropius, Le Corbusier y otros. ¿Por qué? Porque entendieron la excepcional relación entre vivienda y arquitectura. [...] Hoy todo eso ha cambiado. Para la mayor parte de los arquitectos, los encargos más codiciados son los museos y los aeropuertos; ambos se ubican en solares verdes y normalmente libres de valores culturales (1). 
Este interés por la vivienda, unido a un entorno como el peruano en el que era necesario ajustar con precisión los costes y las tecnologías, favoreció la presentación de una serie de propuestas que pretendían ser innovadoras en cuanto a los procesos constructivos. De hecho, en el Plan de Operaciones del PREVI, se hacía referencia expresa a estos requerimientos al describir los propósitos del PP1: Diseño y construcción de un nuevo asentamiento urbano de viviendas de bajo costo, con sus correspondientes facilidades y servicios comunes, basadas en la investigación y desarrollo de soluciones de diseño y tecnología, nuevas o existentes, buscando aumentar la eficacia de los recursos utilizados en la construcción y reducir los costos directos de la misma (2).

Estas premisas incentivaron la presentación de proyectos que planteaban opciones tecnológicas capaces de ser asumidas por la realidad técnica y empresarial del Perú de la época. Se trataba de sistemas constructivos que, lejos de una sofisticación puntera que hubiera resultado inabordable en ese entorno, sí eran capaces de proponer procesos de racionalización y de industrialización de los procesos de obra, así como una reducción de los costes. Se trataba de opciones que pretendían dar respuesta a un amplio sector intermedio afectado por problemas de vivienda en Latinoamérica. Sin embargo, no se dirigían directamente a paliar los casos de pobreza extrema, a aplicarse en situaciones de urgencia o a responder a desastres naturales, ámbitos que dentro del PREVI, encontraban una respuesta más ajustada en el plan PP3, en el que se instaba expresamente a trabajar en ese terreno y a proponer: un sistema de "lotes y servicios" para el asentamiento de familias migrantes y de ocupantes sin títulos (de las tierras tomadas) con muy bajos ingresos (2).
El PREVI quiso ser una oportunidad para construir soluciones viables y económicas que pudieran dar respuesta al problema de la vivienda social latinoamericana. Se proyectaron y construyeron viviendas con sistemas industrializados, que pretendían economizar los costes y reducir los tiempos de obra, y se ensayaron buena parte de las propuestas de industrialización económica de la época. Actualmente, el paso del tiempo y los procesos de ampliación y construcción informal que se han producido sobre las viviendas PREVI, hacen posible evaluar estos sistemas industrializados, no solo desde el punto de vista de su economía o durabilidad, sino también considerando cuáles de ellos han resultado más flexibles y adaptables para los usuarios, o cuáles han convivido mejor con las transformaciones realizadas sobre las viviendas por sus habitantes.

\section{2. ¿QUÉ ES PREVI ACTUALMENTE?}

Una de las características más reseñables del PREVI fue que una buena parte de las propuestas presentadas acabaron materializándose (Figuras 1 y 2). La necesidad de vivienda y la ambición con que se planteó el concurso llevaron a construir la mayoría de las proyectos internacionales presentados. Se encargaron alrededor de 20 viviendas a cada uno de los equipos. A diferencia de las convocatorias en las que se selecciona a una minoría de participantes para que construyan, el carácter experimental y ejecutivo de la iniciativa permitió poner a prueba las distintas opciones que los equipos internacionales barajaban. Ante la gravedad del problema, se entendió que no era posible permitirse el lujo de dejar en el papel las numerosas propuestas de calidad presentadas. El informe final del jurado [...] manifestó el deseo de resaltar la importancia de que se usaran al máximo las diferentes ideas
1. Lima: el barrio PREVI en la actualidad.

Fuente: Elaboración propia a partir de Google Maps.

2. La Unidad Vecinal Experimental del PREVI.

Fuente: Publicación PREVI (2).
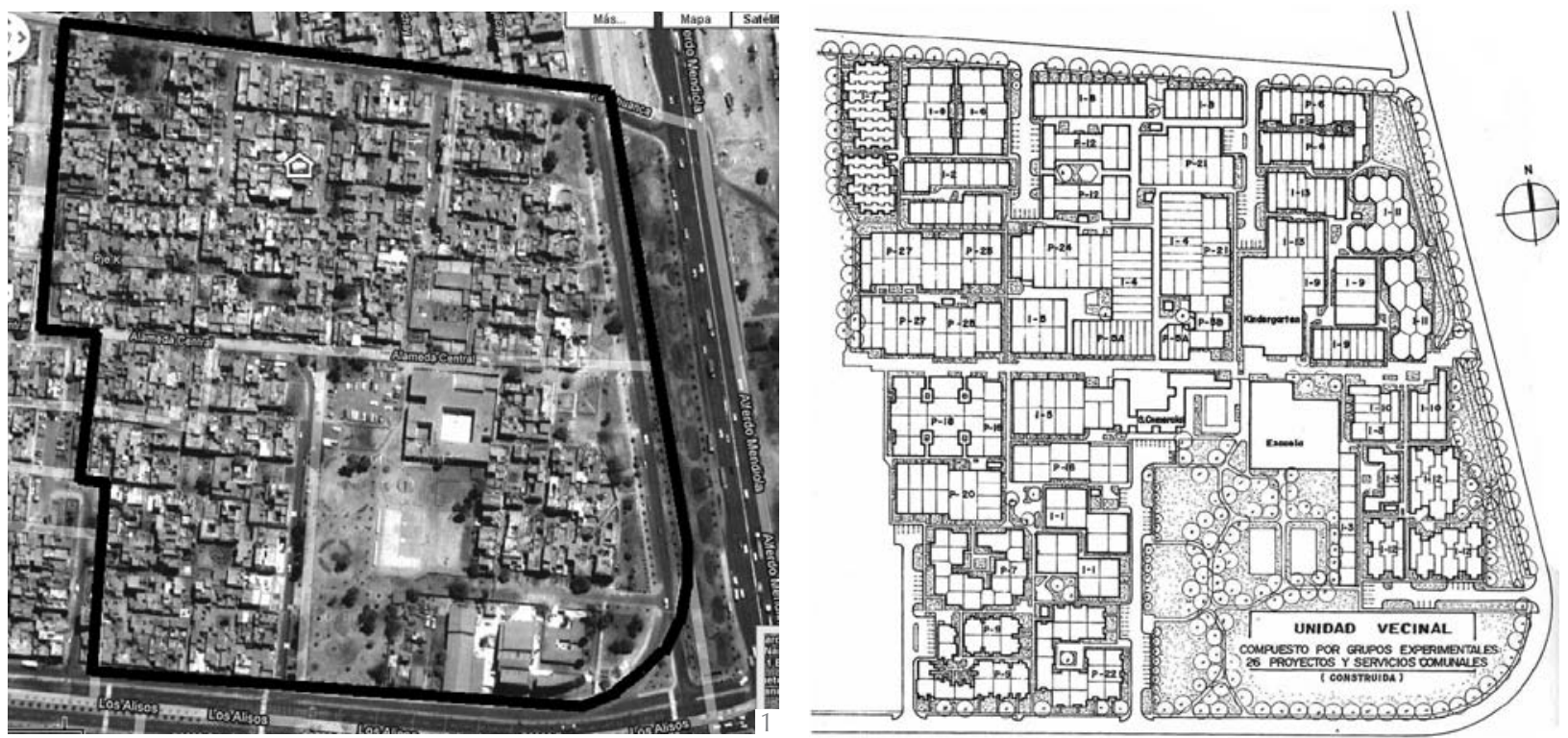
3. Transformación de las viviendas PREVI. Proyectos I11y P7 en su estado original. Estado actual de las propuestas I11, P7, I2, P5, 19, P9, I5 y P21.

4 y 5 . Crecimiento en altura de las viviendas.
6. Mezcla de usos

propuestas por los participantes nacionales e internacionales (2). Este hecho hizo del PREVI un banco de pruebas de técnicas constructivas, y por otra parte, generó un conjunto de viviendas pensadas como tejido urbano que actualmente sigue en uso.

EI PREVI a fecha de hoy es un barrio dentro de la ciudad de Lima (Figura 1), un barrio singular que agrupa los distintos tipos de vivienda proyectados por los arquitectos que participaron en el concurso, pero que lejos de mantenerse al margen del proceso de cambio y modificación de la ciudad de Lima, se ha visto completado, transformado y ampliado por sus usuarios. De esta manera, los proyectos construidos del PREVI permiten ver cuál ha sido la aceptación por parte de los habitantes de las premisas arquitectónicos de partida.
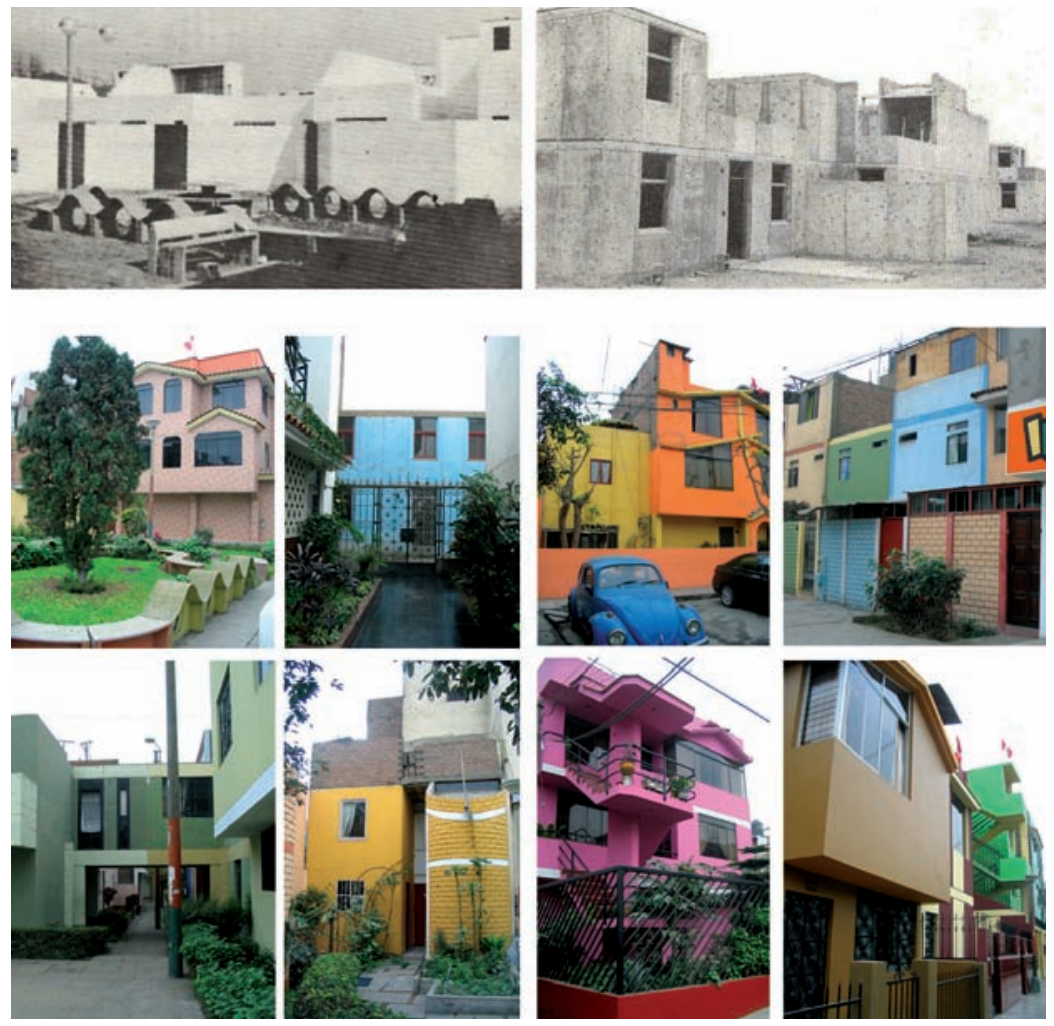

Al examinar los principales cambios que ha sufrido el barrio, salta a la vista el aumento de densidad residencial que se ha operado en el tejido urbano a través de las sucesivas ampliaciones, añadidos y crecimientos en las viviendas (Figura 3). Frente a la opción de los años sesenta, que planteaba una o dos alturas, la realidad apunta al crecimiento vertical (Figura 4 y 5). Las dinámicas urbanas y las ampliaciones realizadas por la mayoría de los habitantes han Ilevado hacia un segmento de ciudad en el que abundan las edificaciones de tres o cuatro plantas y en las que los usos comerciales se mezclan con los residenciales (Figura 6).

De esta manera, el aumento de la densidad edificatoria, la mixtura de usos y la búsqueda de una variedad formal que rompe las series e individualiza las viviendas, son quizá los principales cambios sufridos por los proyectos del PREVI. El uso y el tiempo, a través de las sucesivas ampliaciones, ha efectuado una transformación del proyecto que afecta, no solo individualmente a cada casa, sino también a la agrupación urbana. Los usuarios, a través de sus intervenciones, han interpretado y modificado la propuesta de conjunto, cabría preguntarse ahora si también ha ocurrido lo mismo con la propuesta tecnológica.

\section{RACIONALIZACIÓN CONSTRUCTIVA Y CRECIMIENTO EN EL TIEMPO}

Las bases del concurso instaban a la presentación de propuestas que, a través de la racionalización y la adecuación técnica del diseño, mejorasen los costes y tiempos de obra que proporcionaban las técnicas tradicionales. La apuesta por la innovación estaba clara, pero a la vez había que adecuarla a la realidad limeña de la época, marcada por la migración campo-ciudad y la efervescencia de la construcción suburbial espontánea en los Ilamados pueblos jóvenes, asentamientos informales surgidos a raíz del desborde popular.
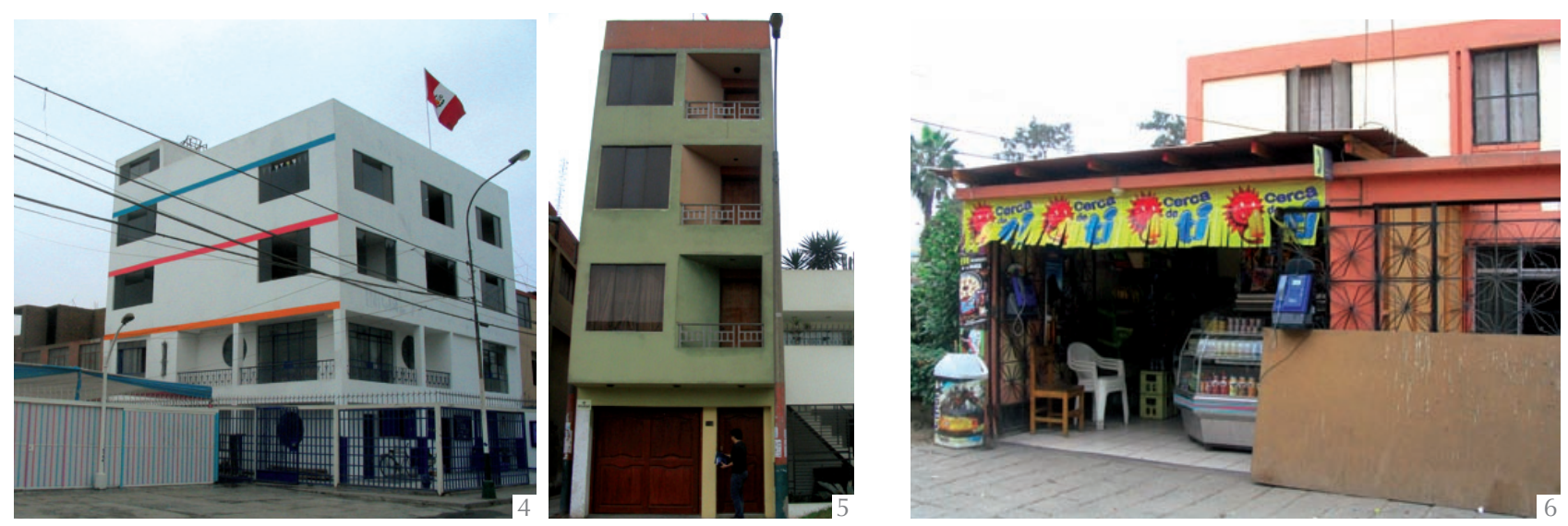
En las acertadas palabras del antropólogo peruano Matos Mar: A las invasiones de tierras en la sierra, acompañan grandes invasiones de predios urbanos en la capital y ciudades principales, dando lugar al crecimiento desmesurado de barriadas y asociaciones vecinales (3). Era necesario contar con que los usuarios, al recibir las viviendas, iban a emprender actividades constructivas de transformación y mejora. De la conjunción entre el deseo de innovación y la realidad local, surgieron las que quizás son dos de las líneas de trabajo más claras dentro del concurso PREVI: la apuesta por la racionalización constructiva y el crecimiento en el tiempo.

\subsection{Vivienda crecedera en el tiempo}

La presión demográfica que la migración desde el campo estaba ejerciendo sobre la ciudad y la aparición de asentamientos espontáneos en los que predominaba la autoconstrucción, hacían pensar que los usuarios de las viviendas podían completarlas y ampliarlas con el tiempo. Los habitantes de las nuevas viviendas habían probado ya, en el sector informal, su capacidad de acción edificatoria. Por ello se plantea a los equipos participantes en el concurso no tanto la realización de un producto acabado, como la construcción de un proceso estructurado desde el que seguir creciendo: Las viviendas serán diseñadas para familias con dos a seis niños, y con posibilidades de expansión para acomodar en el futuro hasta diez personas aproximadamente (2).

Este planteamiento, ya en la temprana fecha de 1968, vendría a cuestionar el entendimiento de la vivienda como producto acabado. La propia necesidad y la atención al entorno local habrían Ilevado a las propuestas a plantearse la vivienda como un proceso constructivo diferido en el tiempo, capaz de adaptarse a las distintas configuraciones familiares de los que, a la vez que habitan la casa, la van transformando.

A este hecho contribuye también la estructura familiar local, que presenta diferencias con respecto a la familiar nuclear tipo para la que suelen proyectarse las promociones de vivienda pública europea. Este tipo de agrupaciones se vería favorecido por la organización económica del sector informal o "contestatario" en palabras de Matos Mar: Las empresas del sector contestatario tienen carácter predominantemente familiar, lo que les da una identidad muy particular. En ellas participan no solo la familia nuclear sino también la extensa (hermanos, tíos, cuñados, primos) (3). La organización familiar extendida, que se podía apreciar entre los destinatarios de las viviendas, habría dado origen a un nuevo tipo de casa, que no respondería ni al esquema de vivienda individual y unifamiliar, ni al de vivienda colectiva sustentada en la idea de propiedad horizontal, es decir del piso.

García-Huidobro denomina multifamiliares a estas viviendas de apariencia individual, pero que albergan a distintos núcleos familiares, generalmente con relaciones de parentesco entre ellos. La vivienda multifamiliar responde a organizaciones familiares diversas, con una complejidad creciente [...] Sin sacrificar la independencia y la calidad de vida, las familias se benefician del ahorro y la eficiencia de la economía de la gran familiar (1).

Es en estas familias ampliadas, además, en las que es más fácil que se produzcan actividades de autoconstrucción que hagan de la vivienda un sistema crecedero. No en vano, la consideración de esta realidad social local ha sido origen de algunas de las propuestas más destacadas dentro del ámbito de la vivienda latinoamericana. En las viviendas galpón del mexicano Carlos González Lobo (4), en las propuestas del chileno Alejando Aravena o en las realizaciones del CEVE argentino, es posible detectar, con distintos matices, esa misma idea de la vivienda semilla, ampliable, mejorable o crecedera en el tiempo.

El estado de transformación en que actualmente se encuentra el PREVI, aunque haya desdibujado por completo muchas de las propuestas formales de los arquitectos, hace evidente la favorable acogida popular de la necesidad del crecimiento. Tal y como se previó, las viviendas se han ampliado. Lo que cabría preguntarse ahora es cómo lo han hecho, por una parte teniendo en cuenta las transformaciones a las que se han sometido los tipos de vivienda y por otra considerando las técnicas constructivas empleadas en las ampliaciones.

\subsection{Racionalización constructiva}

De la misma manera que las bases del concurso instaban a la flexibilidad en el diseño, incentivaban también el empleo de tecnologías con capacidad de adaptación a las transformaciones y cambios. Todos los diseños de las viviendas, así como la tecnología de la construcción, deberán estar basados en el concepto de flexibilidad y de crecimiento progresivos (horizontal y/o vertical), para satisfacer el carácter dinámico y cambiante de las familias que integran la comunidad en sus aspectos social, cultural y económico (2). 
Se buscaban, por tanto, propuestas flexibles y a la vez innovadoras, capaces de reducir costes y tiempos de obra. La intención de mejorar el proceso constructivo dejaba la puerta abierta a las técnicas industrializadas que en ese momento se estaban utilizando en Europa. Pero a la vez, la pretendida flexibilidad y la adaptación a la realidad técnica peruana del momento, hacían necesarias las matizaciones.

Entre los años 50 y 70 la industrialización vivió en los países tecnológicamente más avanzados de Europa un periodo de masividad euforia y negocio: Los sistemas a base de grandes paneles fueron dominantes en la llamada Europa del Este y cuantitativamente muy importantes en los países de la Unión Europea (5). En algunos de los proyectos internacionales presentados al PREVI es posible encontrar todavía muestras de esta confianza en la industrialización pesada. Las propuestas de Polonia y Alemania, dos países en los que se desarrollaron y emplearon estas técnicas, pretendieron transferir a Lima estas prácticas constructivas.

Sin embargo, no será esta la apuesta tecnológica mayoritaria en el concurso. Alrededor del año 1970 se inicia en Europa una nueva etapa para la construcción industrializada, la crisis del petróleo, la reducción del tamaño medio de las obras, y la proporción de viviendas unifamiliares abrieron una etapa de crisis y perplejidad (5). Se empieza a cuestionar entonces la rigidez de los sistemas pesados y su capacidad de adaptación cuando el número de viviendas de cada promoción no es especialmente elevado. Es entonces cuando se empieza a hablar también de los llamados "elementos compatibles".

La crítica a la prefabricación pesada que durante los años setenta se realizaba en Europa, unida a las particularidades de la realidad técnica peruana, Ilevaron a varios participantes a proponer sistemas que más que industriales podríamos denominar como racionalizados. En esta línea se puede situar la propuesta del Tabibloc de Íñiguez de Ozoño y Vázquez de Castro (Figura 8) (6) o los paneles livianos realizados a pie de obra que planteaba el equipo suizo Atelier 5 . Otros equipos, como el formado por los colombianos Esquerra, Sáenz, Urdaneta y Samper, o el holandés, con Aldo van Eyck (Figura 9) a la cabeza, proponían sistemas que racionalizaban las técnicas convencionales empleando bloques de hormigón modulados para formar muros de albañilería armada. Se trataba en ambos casos de ensayar técnicas que mejoraran los procesos tradicionales de construcción, pero que a la vez, no cayeran en la exportación directa de tecnologías incapaces de adaptarse a la realidad limeña.

\section{LAS PROPUESTAS INDUSTRIALIZADAS DEL PP1-PREVI}

La vocación de innovación tecnológica del concurso se convierte en una premisa específica cuando se afronta la ejecución de la Ilamada Unidad Vecinal Experimental, el barrio surgido al construir las propuestas del PP1 del PREVI. Al afrontar la puesta en obra se busca lograr la aplicación a la vivienda social de técnicas constructivas innovadoras dentro de las limitaciones presupuestarias indicadas: El objetivo perseguido fue adaptar y poner en práctica algunos sistemas constructivos de naturaleza experimental propuestos por los concursantes, desarrollar nuevos materiales modulares para los sistemas convencionales, mejorar algunas prácticas y métodos existentes, diseñar y experimentar nuevas soluciones de instalaciones y desarrollar un concepto integral para el equipamiento de los espacios interiores (2).

En un breve repaso a las propuestas de industrialización planteadas en los proyectos originales del PP1 se observa una considerable variedad de soluciones constructivas que, sin embargo, es posible agrupar en tres familias (Tabla 1). Podríamos hablar de proyectos que apuestan por una industrialización pesada a la europea (Figura 7), otros que optan por el empleo de la albañilería racionalizada (Figura 9) y otro grupo de propuestas que abren una interesante vía intermedia que trabaja con procesos racionalizados, basados en la utilización de elementos ligeros más o menos industrializados producidos en pequeñas plantas o a pie de obra (Figura 8).

El proyecto PREVI que quizá ejemplifica de manera más clara las propuestas de industrialización pesada europea, fue el del equipo alemán de Herbert Ohl (Figura 7), que aparece descrito de la siguiente manera: El sistema constructivo propuesto consiste en la yuxtaposición desfasada de grandes módulos o marcos de hormigón armado de 7,20 m. de largo, $3 \mathrm{~m}$. de alto y 1,20 m. de ancho, que se unen por medios mecánicos (pernos o bulones). Cada uno pesa 6 Tn. y es prefabricado en moldes de acero en una planta central cerca de la obra. El montaje se efectúa mediante un puente grúa rodante especial que permite su desplazamiento (7). Propone también la construcción de una escalera de caracol a base de elementos prefabricados tridimensionales.

Como se puede observar en la propuesta alemana, el PREVI surge en un momento en 
Tabla 1

Las tres familias tecnológicas del PP1 del PREVI

\begin{tabular}{|c|c|c|}
\hline \multicolumn{3}{|c|}{$\begin{array}{l}\text { Clasificación de los sistemas constructivos del PP1 del PREVI: } \\
\text { Descripciones de los sistemas constructivos a partir de datos del ININVI (7) }\end{array}$} \\
\hline & $\begin{array}{c}\text { EQUIPOS } \\
\text { INTERNACIONALES }\end{array}$ & $\begin{array}{l}\text { EQUIPOS } \\
\text { PERUANOS }\end{array}$ \\
\hline $\begin{array}{l}\text { PREFABRICACIÓN PESADA } \\
\text { O SEMI-PESADA }\end{array}$ & $\begin{array}{l}\text { - Herbert Ohl (Alemania): Prefabricación pesada } \\
\text { de grandes elementos tridimensionales. } \\
\text {-Oskar Hansen y Svein Hatloy (Polonia): Prefabri- } \\
\text { cación semipesada. } \\
\text { - James Stirling (Reino Unido): Prefabricación en } \\
\text { obra de grandes paneles tipo sandwich. }\end{array}$ & $\begin{array}{l}\text {-Miguel Alvariño: Prefabricación bidimensional } \\
\text { para muros y techos: grandes paneles huecos y } \\
\text { modulados. } \\
\text {-J. Gunther y Elsa Mazzarri-Manuel Llanos: } \\
\text { Prefabricación pesada tridimensional. } \\
\text {-Juan Reiser: Prefabricación de paneles en T } \\
\text { invertida y losa vaciada in situ en la cubierta. }\end{array}$ \\
\hline $\begin{array}{l}\text { PREFABRICACIÓN LIGERA O } \\
\text { RACIONALIZACIÓN } \\
\text { CONSTRUCTIVA }\end{array}$ & $\begin{array}{l}\text {-Atelier } 5 \text { (Suiza): Paneles ligeros prefabricados } \\
\text { a pie de obra que funcionan como encofrado } \\
\text { perdido. } \\
\text {-Íñiguez de Ozono y Vázquez de Castro (España): } \\
\text { Tabibloc: sistema de bloques de hormigón que } \\
\text { funcionan como encofrado perdido con cámara } \\
\text { de aire y conforman prácticamente la vivienda. } \\
\text {-Christopher Alexander (USA): Paneles y viguetas } \\
\text { prefabricados en obra. } \\
\text {-Knud Svenssons (Dinamarca): Hormigón in situ } \\
\text { con encofrados metálicos modulares. }\end{array}$ & $\begin{array}{l}\text {-Ernesto Paredes: Albañilería armada de bloques } \\
\text { de concreto y semiprefabricación liviana en el } \\
\text { techo (Techo Domozed) que reduce en } 40 \% \text { el } \\
\text { costo del techo aligerado convencional. } \\
\text {-C. Morales-E. Montagne: Prefabricación ligera } \\
\text { de módulos de reducido espesor que sirven de } \\
\text { encofrado perdido para el vaciado in situ de } \\
\text { muros, losa canal nervada para la cubierta. } \\
\text {-Williams-Núñez-Miró Quesada, Smirnoff-Ramí- } \\
\text { rez-Wiskowsky-Chaparro y J. Crousse-J. Páez: } \\
\text { Hormigón vertido in situ en muros y techos. No } \\
\text { se trata de prefabricación, sino de racionaliza- } \\
\text { ción constructiva. }\end{array}$ \\
\hline $\begin{array}{l}\text { ALBAÑILERÍA } \\
\text { RACIONALIZADA }\end{array}$ & $\begin{array}{l}\text {-Candilis, Josic and Woods (Francia): Albañilería } \\
\text { armada y viguetas prefabricadas en forjados. } \\
\text {-Germán Samper (Colombia): Albañilería armada } \\
\text { y aligerados de hormigón en forjados. } \\
\text {-Aldo van Eyck (Holanda): Albañilería armada y } \\
\text { módulos huecos de hormigón en forjados. } \\
\text { - Kikutake, Maki, Kurokawa y Noriaki (Japón): } \\
\text { Albañilería armada y bloques de hormigón. } \\
\text {-Charles Correa (India): Albañilería armada a base } \\
\text { de bloques de hormigón. } \\
\text {-Toivo Korhonen (Finlandia): Albañilería armada a } \\
\text { base de bloques de hormigón. }\end{array}$ & $\begin{array}{l}\text { - Eduardo Orrego: Albañilería armada, que en } \\
\text { Perú se aplicó por primera vez en PREVI. } \\
\text { - Luis Vier: Albañilería confinada para muros y } \\
\text { losa aligerada para forjado y cubierta. } \\
\text {-Cooper-García Bryce-Graña-Nicolini: Alba- } \\
\text { ñilería confinada de bloques de hormigón y } \\
\text { techos semiprefabricados de reducida espesor } \\
\text { (U invertida). }\end{array}$ \\
\hline
\end{tabular}

FUENTE: Los autores.

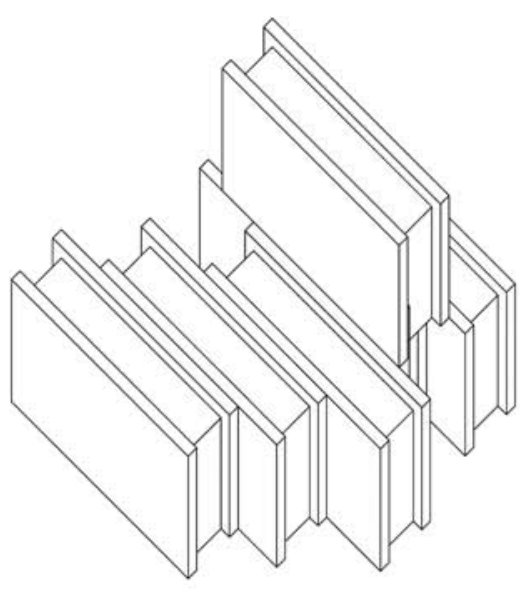

el que todavía la industrialización pesada era la panacea tecnológica del "Norte". Pero el hecho de que en las bases del concurso se hiciera referencia a la posibilidad de que las viviendas fueran ampliadas o modificadas por los propios usuarios, hizo que algunos de los equipos dejaran de lado estas soluciones de grandes elementos tridimensionales, más tecnológicas, pero muy rígidas de cara a posibles modi-
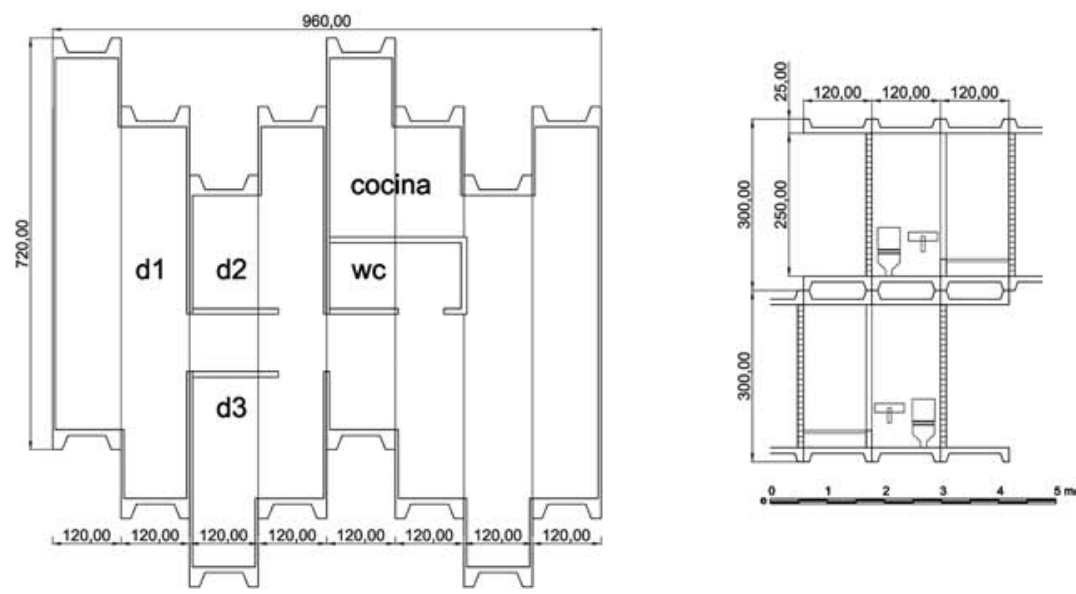

ficaciones. La necesidad de adaptación llevó a algunos participantes a optar por lo que se conoce como albañilería racionalizada, una mejora organizativa de técnicas tradicionales basadas en el empleo intensivo de mano de obra. O bien, a presentar sistemas de prefabricación ligera, o de racionalización de pequeños elementos, que en ocasiones se producían en pequeñas series a pie de obra. 
8. Racionalización constructiva (PREVI-PP1) Arriba: Proyecto 14-Suiza. Arquitecto: Atelier 5. Abajo: Proyecto 19-España. Arquitectos: Íñiguez de Ozoño y Vázquez de Castro.

9. Albañilería racionalizada (PREVI- PP1) Izquierda: Proyecto 111-Holanda. Arquitecto: Aldo van Eyck. Derecha: Proyecto I8-Japón. Arquitectos: Kikutake, Maki, Noriaki y Kurokawa.
Dentro de este tipo de apuestas por la racionalización constructiva puede clasificarse la propuesta del Tabibloc, presentada por el equipo español de Íñiguez de Ozoño y Vázquez de Castro (Figura 8): El sistema constructivo consiste en albañilería confinada realizada con un bloque de hormigón de diseño especial denominado Tabibloc. Cada bloque presenta cuatro tabiquillos que conforman tres espacios interiores, el central que se rellena con mortero, formando una barrera acústica y los laterales que permanecen huecos para mejorar el aislamiento térmico del muro. El Tabibloc presenta 18 variantes de diseño [de bloques] para solucionar intersecciones, encuentros y resolver uniones. En todos los casos funciona como encofrado perdido (7). También la propuesta del equipo suizo Atelier 5 podría servir como ejemplo de este tipo de técnicas constructivas, ya que propone la construcción con paneles prefabricados a pie de obra que funcionan como encofrado perdido (Figura 8).
Estaríamos cerca de los sistemas de racionalización de los que habla Le Corbusier en Hacia una arquitectura cuando describe su proyecto de viviendas para Pessac y dice: Se trata de una verdadera industrialización de la albañilería (8). Curiosamente, en el barrio PREVI como en Pessac, nos encontramos con un tejido residencial compuesto a través de piezas de baja altura, de unidades que se agrupan, pero que no conforman grandes bloques de vivienda colectiva susceptibles de ser construidos a través de elementos pesados. Lejos de las grandes estructuras, estas viviendas no requieren de tecnologías sofisticadas, sino más bien de la planificación de sistemas que racionalicen la construcción a base de elementos ligeros.

Dentro de esta misma línea, aunque más cerca ya de la denominada albañilería racionalizada, es posible encuadrar la propuesta del equipo del holandés Aldo van Eyck (Figura 9): El sistema constructivo

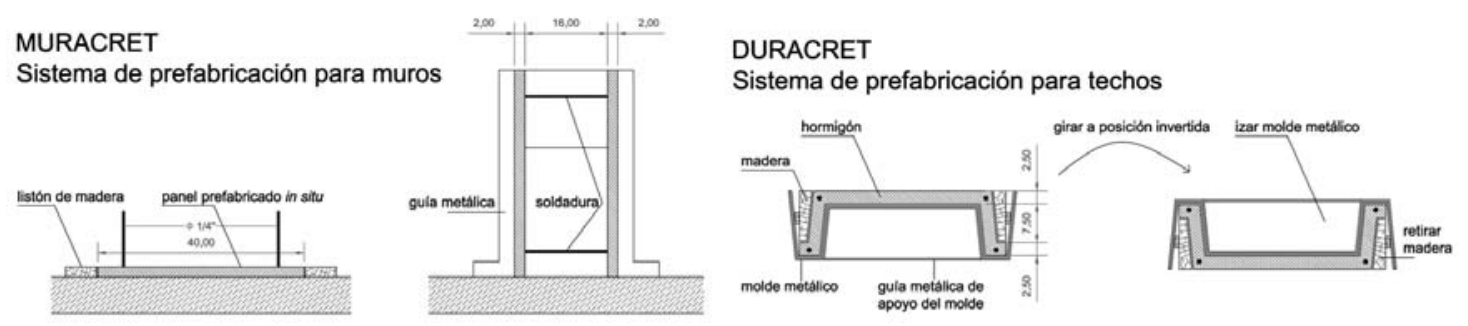

TABIBLOC
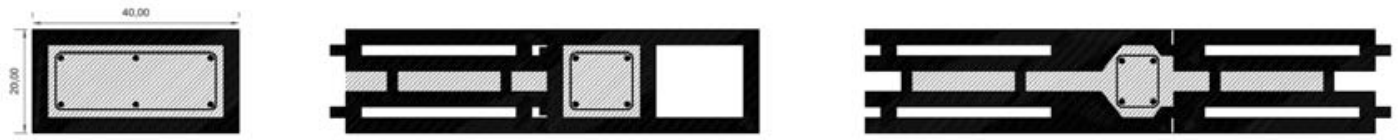

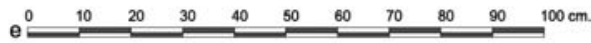

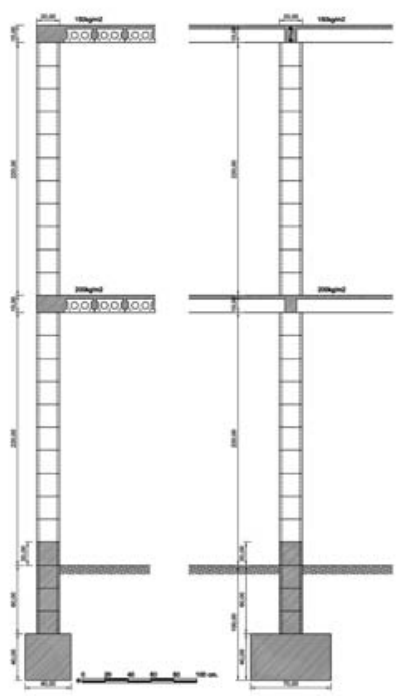

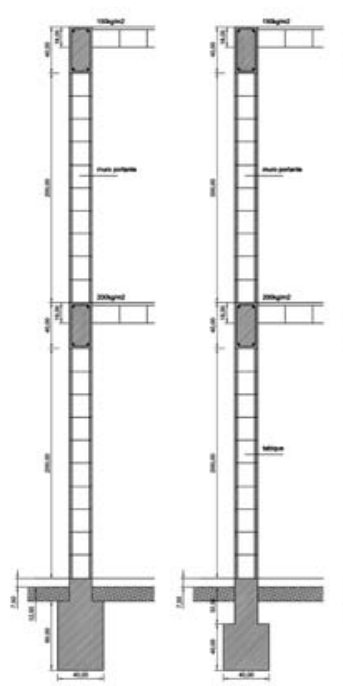

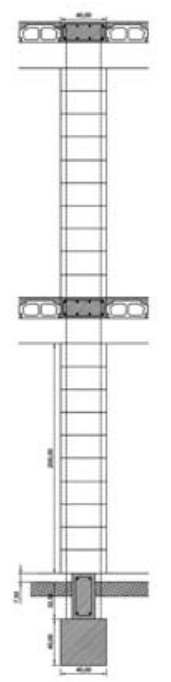

emplea el hormigón y el acero como materiales básicos. Consiste en muros de albañilería armada realizados con bloques de hormigón modulados. La cubierta se realiza con módulos huecos de hormigón armado que cubren las luces entre muros y/o vigas. Se trata de una prefabricación lineal liviana. El nombre de la patente es SKALJA-VITESA (7). También la propuesta del equipo japonés de Kikutake, Maki, Noriaki y Kurokawa, estaría en este mismo grupo. Propone un sistema de albañilería armada con bloques de hormigón tipo PREVI (Figura 9).

En PREVI se proponen distintas innovaciones tecnológicas, pero ¿qué aportaciones y transferencia de resultados se ha producido con el tiempo? A la vista de las sucesivas transformaciones de las viviendas, es posible evaluar la viabilidad real y la interpretación constructiva que han tenido las 
propuestas industrializadas del PREVI, así como plantear la posible transferencia de su uso a otros ámbitos latinoamericanos. El hecho de que las viviendas del PREVI sigan en pleno uso permiten también verificar la respuesta que estos sistemas han dado cuarenta años después de su construcción y además comprobar cuáles han sido las propuestas que han dado mejores resultados cuando los usuarios han decidido ampliar sus viviendas mediante autoconstrucción.

Los proyectos presentados al PREVI aportaron un variado catálogo de soluciones constructivas. En varios casos era posible detectar una apuesta por la racionalización y la prefabricación ligera de pequeños elementos, algunos incluso realizados in situ. Aparte de la adecuación a la realidad local, estas opciones constructivas resultaban también interesantes por el posible germen industrial que podían generar en el tejido empresarial de la zona.

Sin embargo, pasados cuarenta años que evidencian que la construcción incremental no ha parado en el barrio, es difícil detectar el arraigo de las propuestas tecnológicas en el tejido empresarial local. La mayor parte de las ampliaciones y crecimientos de las viviendas se han realizado empleando técnicas nítidamente tradicionales. ¿Significa esto que las aportaciones tecnológicas del PREVI no fueron aprovechadas? Si por una parte parece que no arraigaron en el mercado local, por otra sí es posible detectar la relación de las tecnologías propuestas en el PREVI con otras técnicas constructivas empleadas en Latinoamérica.

Sistemas como el Sandino (Cuba), el Bloquepanel (Costa Rica), Servivienda (Colombia) o el Sancocho (Venezuela) (9), basados en la prefabricación racionalizada de pequeños elementos, podrían englobarse dentro de la misma familia constructiva que algunos de los sistemas PREVI. Parece posible deducir que el germen industrial que intentó generar el PREVI en su tiempo, es una semilla tecnológica que, desde distintos ámbitos, sigue intentando arraigar en Latinoamérica. Sin embargo, parece que la realidad tecnológica que genera el mercado es otra.

\section{ALGUNAS CONSIDERACIONES ECONÓMICAS}

A la hora de analizar tecnologías de bajo coste destinadas a la construcción de vivienda social no es pertinente ceñirse únicamente a los aspectos técnicos, sino que por la propia formulación del problema, se hace necesario atender también cuestiones de gestión económica. Por eso, al evaluar las tecnologías del PREVI y pensar en su posible vigencia en la actualidad, es preciso preguntarse por las condiciones sociales y económicas en que se desarrolló el concurso.

El PREVI surge como iniciativa de Fernando Belaúnde Terry, arquitecto y presidente de Perú entre los años 1963 y 1968 y se plantea como una propuesta de vivienda social en la que el Estado aparece implicado. Por otra parte, hay que señalar también la participación activa de Naciones Unidas junto al gobierno peruano apoyando la iniciativa. Distintos avatares políticos llevaron a un cambio de gobierno, pero la idea inicial de la propuesta se mantuvo, se trataba de un proyecto de vivienda con participación pública. A fecha de hoy, una de las primeras cuestiones que es necesario plantearse es si esta determinación política inicial sigue vigente, si la vivienda se sigue entendiendo hoy como una tarea del Estado o si más bien se ha convertido en un ámbito de inversión de mercado.

Por otra parte, como casi siempre que se invoca a la industrialización de la construcción, aparece el problema laboral. EI PREVI contemplaba la posibilidad de la construcción participativa, un supuesto que habría que comprobar si sigue vigente en nuestros días. Además, a la hora de emprender proyectos de vivienda industrializada, se hace necesario considerar también la influencia que las distintas propuestas tecnológicas tienen sobre el empleo. La espinosa cuestión de la creación de puestos de trabajo, que estos procesos industriales tienden a reducir, ha sido en muchos casos un freno para su implantación; puesto que se han interpretado como generadores de desempleo, más que como una oportunidad para fomentar la creación de puestos de trabajo más cualificados y con mejores condiciones laborales.

Por tanto, a la hora de plantear soluciones de vivienda industrializada no solo habría que considerar la posibilidad de transferir unas determinadas prácticas constructivas, sino también verificar si la situación de desarrollo industrial y económico que esas técnicas implican, suponen una opción social y económicamente viable en el país donde se implantan. En el caso de la vivienda protegida es preciso, además, verificar la capacidad adquisitiva de la población a la que se dirigen y adaptándose a esta situación, plantear soluciones constructivas que se muevan en un rango de precios que las haga viables. A la hora de evaluar las tecnologías del PREVI y su posible vigencia actual, habría que preguntarse también, dentro de lo económico, quiénes fueron los destinatarios de esas 
${ }^{1}$ El quintil de ingreso se calcula ordenando la población de mayor a menor según sus ingresos y dividiéndola en cinco grupos o tramos, de manera que el primer quintil agrupa a la quinta parte de la población más pobre y el quinto a la más rica.

10. Grupo social al que se destinaba el PREVI.

Fuente: Publicación PREVI (2). viviendas y quiénes podrían serlo ahora en Perú y en otros países latinoamericanos.

\section{1. ¿A qué sector económico se dirigían las propuestas PREVI?}

Los proyectos construidos del PP1 del PREVI se destinaron a familias de "escasos recursos, pero con cierta capacidad adquisitiva". En lo que respecta al costo de las viviendas, la comunidad pretende abarcar una sección representativa de familias de ingresos bajos, pero con posibilidades de destinar un porcentaje de los mismos para la adquisición y financiación de sus viviendas (2) (Figura 10). Estaríamos, por tanto, lejos de los niveles de pobreza extrema hacia los que se dirigiría la propuesta del PP3 de sitios y servicios.

Las familias a las que se dirige el PP1 del PREVI, a pesar de contar con ciertos recursos, no tenían fácil el acceso a la vivienda de mercado. La documentación oficial caracteriza así a este grupo social: El sector al cual se quiere atender (en el año 1968) abarcará las familias con ingresos familiares de aproximadamente 2,800 a 5,800 soles mensuales. Sin embargo, se explica entre paréntesis que, debido a la situación económica, este propósito inicial varió a lo largo del tiempo. (En el desarrollo posterior del proyecto variaron los costos de construcción, los ingresos familiares y las condiciones de financiamiento, haciendo necesaria una actualización permanente de precios hasta el momento de adjudicación de las viviendas) (2).

En un principio, los proyectos del PREVI estaban dirigidos a un sector socio-económico diferente del aue posteriormente ocupó las

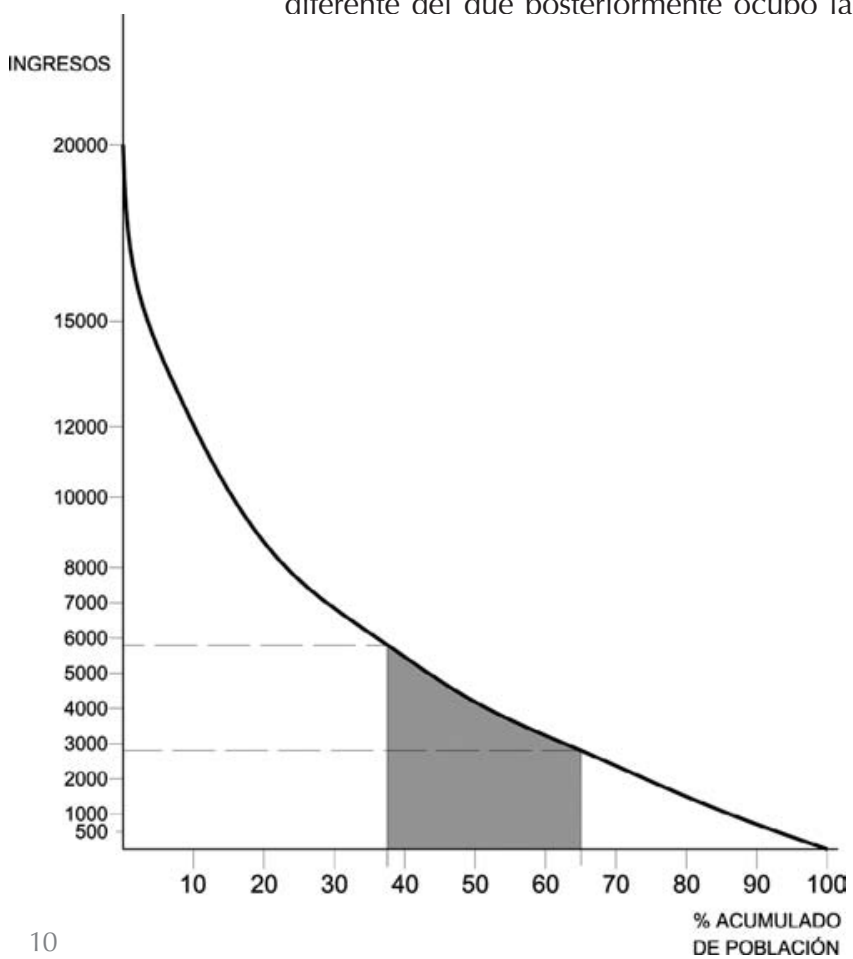

viviendas. Cuando se plantea el concurso las zonas cercanas al área del PREVI estaban siendo ocupadas por migrantes y familias locales de escasos recursos, pero con capacidad de construcción progresiva en el tiempo. Se pensaba que PREVI sería el ejemplo a seguir. Sin embargo, las familias que se instalaron en PREVI, once años después, estarían más cerca de la llamada clase media: empleados bancarios, profesionales independientes, funcionarios de la administración pública..., un grupo social que, aunque vio mermada su capacidad adquisitiva por la crisis, no perdió la oportunidad de hacer crecer informalmente sus viviendas. Por otra parte, durante la época del gobierno militar, no hubo especial interés en promover los resultados del PREVI y dar asistencia técnica a aquellos que quisieran ampliar las viviendas, lo que probablemente contribuyó también al empleo de técnicas constructivas tradicionales en los crecimientos sucesivos.

\section{2. ¿A qué sector se dirigiría hoy?}

Para extraer posibles enseñanzas y aplicaciones actuales de las propuestas del PREVI, un proyecto experimental de vivienda realizado en los años 70, parece conveniente apuntar algunos datos sobre la situación económica peruana actual, al menos en lo que se refiere a los porcentajes de distribución del ingreso nacional por quintiles ${ }^{1}$ (Tabla 2 ).

Tabla 2

Perú: Porcentajes de distribución del ingreso nacional por quintiles de población

\begin{tabular}{|c|c|}
\hline \multicolumn{2}{|l|}{ Año 2008} \\
\hline $\begin{array}{l}\text { PIB por habitante } \\
\text { (a precios corrientes } \\
\text { de mercado) } \\
\text { (\$ USA) }\end{array}$ & $4.471,30$ \\
\hline$\%$ de ingreso $5^{\circ}$ quintil & 52,09 \\
\hline$\%$ de ingreso $4^{\circ}$ quintil & 21,65 \\
\hline$\%$ de ingreso $3^{\circ}$ quintil & 13,73 \\
\hline$\%$ de ingreso $2^{\circ}$ quintil & 8,50 \\
\hline$\%$ de ingreso $1^{\circ}$ quintil & 4,03 \\
\hline
\end{tabular}

Como ocurre en otros países latinoamericanos, se constata que más de la mitad del ingreso peruano lo acapara el quinto quintil, mientras que el $48 \%$ restante se distribuye entre el $80 \%$ de la población, de manera que dos quintas partes de los peruanos, los que se encuentran en el primer y segundo quintil, disponen tan solo de un 12,53\% del ingreso nacional. Este reparto tan diferenciado tiene su reflejo en el sector de la construcción. El quintil más favorecido, debido a su nivel de ingresos, puede generar un mercado para viviendas equiparables a las europeas que, sin embargo, se muestran como soluciones inaccesibles para la 
mayor parte de la población. Este hecho ayudaría a explicar la falta de arraigo de las soluciones industrializadas tipo PREVI en el tejido empresarial peruano.

Puede ser de interés intentar una posible relación entre el porcentaje de consumo del ingreso con el coste de la vivienda en número de salarios por metro cuadrado ${ }^{2}$, y relacionar estos datos con tres hipotéticos sectores que operan en el mercado de la construcción y que denominamos como: libre, oficial e informal. El resultado que se obtiene apunta en la dirección señalada antes. Tan solo el quintil más favorecido responde a las características económicas del sector libre de la construcción. Los dos quintiles siguientes podrían responder a los presupuestos de un sector oficial en el que la vivienda contase con algún tipo de ayuda por parte de los poderes públicos; y los dos quintiles más desfavorecidos que estarían dentro de los parámetros que rigen en los sectores informales (Figura 11).

Ante esta situación económica, soluciones como las que se barajaron en el PREVI podrían hipotéticamente dar respuesta a la demanda de vivienda de los sectores sociales situados alrededor del tercer y cuarto quintil, aunque lamentablemente dejarían fuera de su ámbito de aplicación al sector más desfavorecido, el último quintil, cuya situación económica parece responder más a propuestas del tipo sitios y servicios. De hecho, en el propio PREVI, junto a los proyectos de vivienda, se llevó a cabo la redacción de un proyecto de este tipo, el PP3 que ya hemos mencionado antes.

\section{3. ¿Serían viables las soluciones PREVI en otros países latinoamericanos?}

Atendiendo a la distribución del ingreso por quintiles puede constatarse que la situación de Perú es bastante similar a la de otros países latinoamericanos. Comparando los datos actuales (enero 2010) con los de Brasil y Venezuela, que presentan respectivamente el mayor y el menor ingreso porcentual para el primer quintil, la situación peruana se presenta como intermedia entre dos extremos que no están significativamente distantes entre sí, lo que nos lleva a pensar en la viabilidad de las soluciones PREVI para otros países latinoamericanos (Figura 12).

La superposición de las curvas de ingresos por quintiles en distintos países de la zona arroja un panorama económico en el que, a pesar de las singularidades nacionales, el reparto sigue unas tendencias uniformes, lo que hace factible pensar en la posibilidad de aplicar soluciones similares para sectores sociales en situaciones análogas en otros países latinoamericanos (Figura 13).

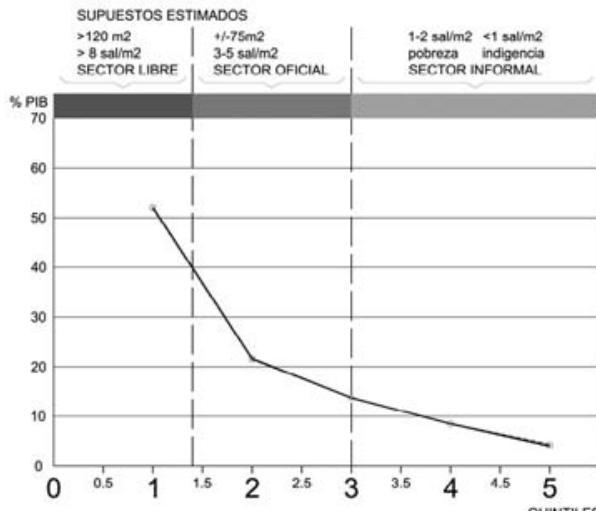

11. Perú: Distribución del ingreso en relación con los precios de la construcción en salarios $/ \mathrm{m}^{2}$. Datos: Anuario estadístico de América Latina y el Caribe 2009, CEPAL (10).
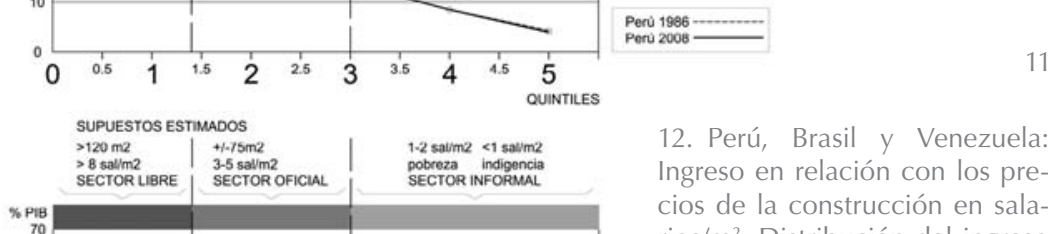

12. Perú, Brasil y Venezuela: Ingreso en relación con los precios de la construcción en salarios $/ \mathrm{m}^{2}$. Distribución del ingreso por quintiles en relación con los precios de la construcción en salarios $/ \mathrm{m}^{2}$.

Datos: Anuario estadístico de América Latina y el Caribe 2009, CEPAL (10).
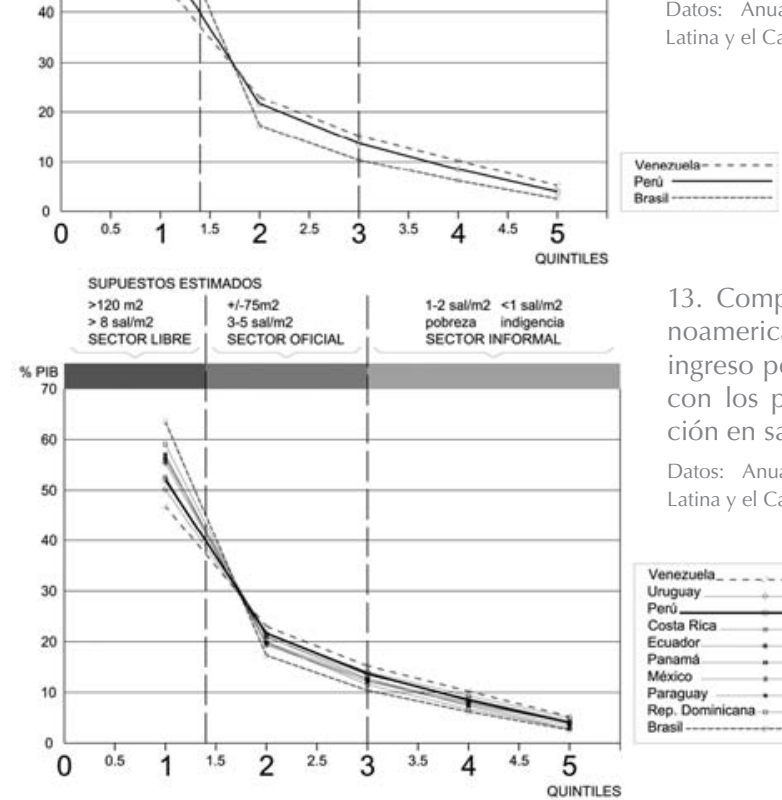

13. Comparativa de países latinoamericanos: Distribución del ingreso por quintiles en relación con los precios de la construcción en salarios $/ \mathrm{m}^{2}$.

Datos: Anuario estadístico de América Latina y el Caribe 2009, CEPAL (10).

\section{CONCLUSIONES}

Cuarenta años después de la construcción de las propuestas del PREVI, parece posible extraer varias conclusiones en relación con el propio concurso y con las tecnologías constructivas que se presentaron, también considerar el resultado que estas han dado, de cara a plantear su posible vigencia para la construcción de nuevas viviendas en Latinoamérica.

La primera cuestión a evaluar sería el planteamiento del propio PREVI y de las que se han considerado dos de sus apuestas básicas: el crecimiento en el tiempo y la racionalización constructiva. En el caso de la primera propuesta cabría señalar el acierto del concurso al incorporar ya en sus bases la llamada de atención hacia la que fue, y sigue siendo, una situación típica de la realidad latinoamericana. Valorar las posibilidades de ampliación y plantear esta cuestión a los arquitectos
${ }^{2}$ El modelo de análisis está tomado de: SALAS, J., coord. Iberoamérica ante HABITAT II. Actas de las jornadas celebradas en la Casa de América, CYTED, Ministerio de Fomento, Madrid, 1996 (11). 
invitados, contribuyó en buena medida a que estos plantearan tipos de viviendas susceptibles de crecer de manera adecuada, evitando en lo posible la generación de estancias mal iluminadas y ventiladas.

En lo que respecta al otro eje del planteamiento del concurso, la apuesta por la innovación técnica, cabría señalar el acierto en lo que se refiere a promover técnicas de racionalización constructiva y prefabricación de elementos ligeros. Apostar por la innovación y a la vez solicitar flexibilidad, llevó a algunos equipos, como el de Vázquez de Castro y Ozoño, Samper, Aldo van Eyck o Atelier 5, a presentar propuestas que se movían en un grado intermedio de industrialización. Se proponía en estos casos la mejora de la organización del proceso constructivo y la realización de piezas seriadas en pequeñas plantas de producción intensivas en mano de obra y selectivas en bienes de capital (5) o incluso a pie de obra. Este camino intermedio entre la industrialización importada y la construcción tradicional, parece a fecha de hoy una de las vías con más posibilidades en Latinoamérica.

Por otra parte, no es casual que fueran precisamente los equipos de Alemania, Polonia y Reino Unido, países con un alto grado de desarrollo tecnológico, los que presentaran soluciones prefabricadas con elementos pesados. Otra de las conclusiones que es posible extraer del PREVI es la relación directa de las tecnologías con las condiciones sociales y económicas del lugar en el que se gestaron, los genes tecnológicos en expresión de K. Reddy (12).

El hecho de que estas condiciones no sean fácilmente exportables, hace evidente la atención que hay que prestar a la evaluación de las capacidades técnicas reales del lugar en el que van a construirse los proyectos, especialmente cuando se trata de proyectos de cooperación. No parece posible plantear la prefabricación de elementos cuyo peso o volumen sean incapaces de mover las personas y/o las maquinarias locales simples. De ser así, los proyectos se convertirán en propuestas inviables, a menos que también se pretendan exportar a esos países patentes y equipos, con lo que probablemente no se conseguiría otra cosa que acrecentar su dependencia tecnológica y en consecuencia económica.

A la vista de las propuestas del PREVI se puede evaluar de manera positiva la capacidad de adaptación de la industrialización ligera y de los procesos de racionalización constructiva, pero al observar las modificaciones realizadas en los proyectos y comprobar que se han realizado mayoritariamente con técnicas tradicionales, parece obligatorio preguntarse por qué estas tecnologías no han arraigado en el tejido industrial local.

Es aquí donde quizá sea necesario apelar a motivos económicos. Parece probable que el sector de la construcción se haya movido de acuerdo con las necesidades de los quintiles que mueven la mayor parte del ingreso y que se haya dejado de lado la posible implementación de tecnologías que en un primer momento estaban enfocadas hacia otros grupos sociales. Actualmente, Perú se encuentra en un momento de crecimiento económico gracias al desarrollo de un importante sector agrícola exportador y parece previsible el aumento en la demanda de viviendas. Las nuevas condiciones podrían hacer viable considerar experiencias como el PREVI y considerar sus aplicaciones no solo en Perú, sino posiblemente también en otros ámbitos latinoamericanos.

\section{BIBLIOGRAFÍA}

(1) García-Huidobro, F.; Torres Torriti, D. y Tugás, N.: ;El tiempo construye! El Proyecto Experimental de Vivienda (PREVI) de Lima: génesis y desenlace, Editorial Gustavo Gili, Barcelona, 2008.

(2) Barrionuevo, R.; Jara, C. y Santolalla, J.: Publicación PREVI, edición del Ministerio de Vivienda y Construcción del Perú, Lima, 1971.

(3) Matos Mar, J.: Desborde popular y crisis del Estado. Veinte años después, Fondo editorial del Congreso del Perú, Lima, 2004.

(4) González Lobo, C.: Vivienda y ciudad posibles, Editorial Escala, Bogotá, 1999.

(5) Salas Serrano, J.: La industrialización posible de la vivienda latinoamericana, Editorial Escala, Bogotá, 2000.

(6) Vázquez de Castro, J. A.: Sistema integral Tabibloc, serie 20 de industrialización de la construcción: prefabricación ligera, Imprenta Safer, Madrid, 1981.

(7) ININVI: PREVI 20 años después: resultados y conclusiones, edición de la Secretaría Ejecutiva del Programa de Actividades PREVI'88, ININVI, Lima, 1988.

(8) Le Corbusier: Hacia una arquitectura, Ediciones Apóstrofe, Barcelona, 1977.

(9) Lorenzo Gálligo, P. (coord.): Un techo para vivir. Tecnologías para viviendas de producción social en América Latina, CYTED, subprograma XIV, proyecto XIV.3 y XIV.5, Edicions UPC, Barcelona, 2005.

(10) CEPAL: Anuario estadístico de América Latina y el Caribe 2009, CEPAL, ONU, 2010.

(11) Salas Serrano, J. (coord.): Iberoamérica ante HABITAT II. Actas de las jornadas celebradas en la Casa de América, CYTED, Ministerio de Fomento, Madrid, 1996.

(12) Reddy, K.: Background and Concept of Appropiate Technology, UNIDO, Conferencia en India, Nueva Delhi, 1998 